Decision Strategies while Intoxicated relate to Alcohol-Impaired Driving Attitudes and Intentions

Sara D. McMullin ${ }^{1}$, Courtney Motschman ${ }^{1}$, Laura Hatz ${ }^{1}$, Denis McCarthy ${ }^{1}$, Clintin P. Davis-Stober ${ }^{1}$

${ }^{1}$ Department of Psychological Sciences, University of Missouri - Columbia, Columbia, Missouri, USA

Corresponding Author: Denis McCarthy (․mccarthydm@missouri.edu) 


\begin{abstract}
Objective: Approximately 28 million individuals engage in alcohol-impaired driving (AID) every year. This study investigated individuals' AID decision making strategies under intoxication, their variability across the breath alcohol concentration curve (BrAC), and the association between strategy and AID attitudes and intentions. Method: 79 adults (23.9 years, $57 \%$ women) who drank alcohol $\geq 2$ days per week and lived $>2$ miles away from their typical drinking locations completed an alcohol administration protocol and AID decision making task. AID attitudes, intentions, and behaviors were assessed repeatedly across the BrAC curve. Bayesian cognitive modeling identified decision strategies used by individuals on the AID decision making task, revealing whether alcohol consumption level and/or ride service cost factored into individuals' decisions to drive while impaired or obtain a ride. Additional analyses tested whether AID attitudes and intentions were related to individuals' decision strategies. Results: Two decision strategies were examined on the ascending and descending limb of the BrAC curve: compensatory (both consumption level and ride service cost factored into AID decisions) and non-compensatory (only consumption level factored into AID decisions). Switching to a compensatory strategy on the descending limb was associated with lower perceived intoxication, perceiving AID as less dangerous, and being willing to drive above the legal BrAC limit. Conclusions: Results suggest that risk for engaging in AID is higher for those using a cost-sensitive, compensatory strategy when making AID decisions under intoxication. Future research is needed to test whether AID countermeasures (e.g., subsidized ride services) are differentially effective according to decision strategy type. Keywords: alcohol, decision making, cognitive modeling, intoxication, driving
\end{abstract}




\section{Public Health Significance}

This study indicates that being cost-sensitive to ride service prices later in a drinking episode is a riskier decision strategy and is related to riskier alcohol-impaired driving attitudes and intentions.

\section{Funding Acknowledgments}

This research was funded by the National Institute on Alcohol Abuse and Alcoholism (NIAAA) and the Office of Behavioral and Social Sciences Research (OBSSR) R01AA019546 (PI: Denis McCarthy) and supported by K25AA024182 (PI: Clintin Davis-Stober) and T32AA013526 (PI: Kenneth Sher). 


\section{Introduction}

Alcohol-impaired driving (AID) is a significant public health issue in the United States, accounting for approximately 28\% of fatal car crashes in 2019 (NHTSA, 2020). Despite overwhelmingly negative perceptions of AID and widespread understanding of its risks (Drew et al., 2008), 28 million individuals in the U.S. engage in AID each year (NHTSA, 2020), resulting in approximately 111 million AID episodes (CDC, 2015). Factors that increase the likelihood someone will decide to drive impaired include personality traits (Jonah, 1997; Treloar et al., 2012), risk perceptions (Sloan et al., 2017; Gustin \& Simons, 2008; Greenfield \& Rogers, 1999), and environmental contexts (Greene et al., 2018; Motschman et al., 2020a; Motschman et al., 2020b).

Understanding the use and barriers to alternative transportation methods (e.g., ride service, designated driver, public transportation) is a critical factor in preventing AID events. Perceived acceptability of AID alternatives by the general public predicts AID behavior (Fairlie et al., 2010; Turrisi \& Jaccard, 1992), and facilitating the use of alternatives has long been a part of AID prevention efforts (see Fell et al., 2020 for a review). Ride service companies (e.g., Uber, Lyft) often claim that they reduce AID through decreasing the associated costs and increasing the convenience of securing a ride after drinking (Uber \& MADD, 2015), and recent data provide some support for this argument (Friedman et al., 2020; Conner et al., 2021). Though ride service companies often offer a lower-cost AID alternative, the monetary cost of ride services may still present a barrier that influences its use (McCarthy et al., 2021). While the type and cost of AID alternatives vary across circumstances, ultimately, all AID events result from an individual choosing to drive rather than seeking an alternative. 
In a recent study, we developed a modeling framework and associated decision task to examine the process by which individuals make decisions about driving or using a ride service after consuming alcohol (McCarthy et al., 2021). Individuals were presented with a series of hypothetical scenarios that systematically varied the number of drinks consumed (in a 2-hour period) and the cost of a ride service alternative. Individuals were asked to choose whether they would drive or pay for the ride service based on each hypothetical scenario. Bayesian cognitive modeling was used to identify the strategy individuals used to make their decisions on the task. Most participants $(\approx 80 \%)$ were classified as using either a compensatory strategy, integrating both drink number and ride costs into their AID decisions, or a non-compensatory strategy, where one or more decision factors were ignored when making AID decisions. Noncompensatory decision makers made AID decisions based only on hypothetical alcohol consumption level, ignoring ride service cost after a set consumption level was reached. Compensatory decision makers, in contrast, factored both hypothetical consumption and ride costs into their AID decisions. Importantly, compensatory strategy users reported higher frequency of recent AID (McCarthy et al., 2021), linking behavior on the decision task to selfreported AID behavior.

Prior research on decision making has investigated individual differences in compensatory and non-compensatory strategy use across myriad environments and applications (Gigerenzer \& Goldstein, 1996; Lee et al., 2019). Much of the research has focused on ethical (Mecca et al., 2014), financial (Newell et al., 2003), and medical decisions (Von Gunten \& Scherer, 2019). Von Gunten and Scherer (2019) found that individuals more often use noncompensatory strategies when asked to make medical decisions for others and compensatory strategies when making medical decisions for themselves. However, other than McCarthy et al. 
(2021), this conceptual framework has not been used to examine alcohol-related decision processes at the individual-level.

A limitation of McCarthy et al. (2021) is that participants completed the decision task while sober. Although individuals can take preparatory steps prior to consuming alcohol to avoid AID (e.g., securing a designated driver, using a ride service), ultimately, the choice to drive while impaired by alcohol is made while impaired by alcohol. The acute effects of alcohol are known to alter cognition and decision making processes relevant to AID decisions (Obst et al., 2018; Weafer \& Fillmore, 2012; George, Rogers, \& Duka, 2005; van Ravenzwaaij et al., 2012). Additionally, studies have found that even a moderate dose of alcohol alters AID attitudes and intentions (Ouimet et al., 2020; Amlung et al., 2014; Motschman et al., 2020a; Motschman et al., 2020b). Therefore, it is critical to understand whether the acute effects of alcohol influence decision making strategies concerning AID decisions.

The goal of the current study was to further investigate the ecological validity of the McCarthy et al. (2021) AID decision task, and subsequent strategy classifications, under acute alcohol intoxication. Participants completed the decision task twice in each session, corresponding to assessment on the ascending (i.e., while breath alcohol concentration $[\mathrm{BrAC}]$ is rising) and descending (i.e., while BrAC is decreasing) limb of the BrAC curve, allowing us to test whether individuals were stable in their strategy use or switched strategies across limbs. Previous research has observed differences in cognition and subjective intoxication across the ascending and descending limbs (Schweizer et al., 2004; 2008) and this discrepancy appears to be particularly important for AID decision making (Motschman et al., 2020a; 2020b). Importantly, we tested how AID decision strategies, and the acute effects of alcohol on decision 
strategies, were related to AID attitudes, intentions to drive above the legal limit, and AID behavior to discern the potential implications of intoxicated decision strategies on AID risk.

\section{Method}

\section{Participants}

Participants were, on average, young adults $\left(\mathrm{M}[\mathrm{SD}]_{\mathrm{age}}=23.9[3.18]\right)$, mostly women (57\%), and mostly white $(83.5 \%, 10.1 \%$ Black, 3.8\% Asian, $2.6 \%$ Other) with an average income between $\$ 15,000$ - \$25,000. Participants were recruited for an "alcohol and decision making study" via advertisements posted at Columbia, MO businesses and emailed via University of Missouri listservs. Participants completed the laboratory alcohol administration protocol described below as well as a larger study protocol involving follow-up ecological momentary assessment not relevant to the analyses presented. Participants were required to be 21 to 35 years of age, report typical alcohol consumption of at least two days per week, have consumed $\geq 4$ (women) or $\geq 5$ (men) standard drinks on at least one occasion over the previous 6 months, have a BMI of 18 to 35 , have access to a motor vehicle and drive regularly, and live at least 2 miles away from their typical drinking locations. Participants were excluded if they were pregnant or nursing, taking medication(s) contraindicated to consuming alcohol, or reported a medical, psychiatric, or substance use disorder. The sample included 79 participants who completed the alcohol administration session.

\section{Measures}

\section{Baseline Measures}

AID Attitudes. Participants reported on their baseline attitudes toward driving after drinking with a single item, "How dangerous do you think it is to drive within 2 hours after 
having 3 drinks?" (Grube \& Voas, 1996). Participants rated responses using a Likert scale from 1 $=$ "not at all dangerous" to $4=$ "very dangerous."

AID Behavior. Participants reported the number of times they had driven in the past 3 months and 1 year after consuming 3 or more drinks within a 2 hour period.

\section{Measures Administered under Intoxication}

Breath Alcohol Concentration (BrAC). An FST-Alco-Sensor breathalyzer

(Intoximeters, Inc., St. Louis, MO, USA) was used to assess BrAC. BrAC was transformed to an interpretable scale by multiplying raw values by 100 so that slope values corresponded to $0.01 \%$ increases in BrAC from the mean (Motschman et al., 2020b).

Subjective Intoxication. Participants reported on their levels of subjective intoxication with a single item, "How intoxicated do you feel right now?" Participants responded using a slider bar on a visual analogue scale (VAS) from $0=$ "not at all" to $100=$ "extremely."

Intoxicated Perceived Danger. Participants reported on how dangerous they perceived it would be for them to drive with a single item, "How dangerous do you feel it is for you to drive right now?" Participants responded using a slider bar on a VAS from $0=$ "not at all" to $100=$ "extremely" (Amlung, Morris, \& McCarthy, 2014).

Intoxicated Driving Intentions. Participants reported on their willingness to drive with three items assessing, "Would you drive a distance of _ mile(s) right now?" Participants responded dichotomously "yes" or "no" whether they would drive distances of 1, 3, and 10 miles. We used participants' BrAC data and this set of intoxicated driving intentions responses to calculate the maximum $\mathrm{BrAC}$ at which participants were willing to drive at least 1 mile (maximum BrAC willing to drive). 
Intoxicated Driving Decision Making Task. A computerized task displayed hypothetical drinking scenarios to participants (see McCarthy et al., 2021). On each trial, participants were asked to decide whether they would choose to drive or seek a safe ride home using a taxi or ride service based on the scenario provided. Scenarios systematically varied in the number of (hypothetical) drinks consumed across 5 different levels, ranging from 2 to 6 drinks in 1-drink increments $(2,3,4,5$, and 6 drinks). Scenarios also systematically varied in the costs (USD) to secure a ride service, ranging from $\$ 5$ to $\$ 25$ in $\$ 5$ increments $(\$ 5, \$ 10, \$ 15, \$ 20$ and \$25). The cost to drive was always $\$ 0$. Each drink and cost level were paired and produced 25 total combinations of drink quantities and ride costs ( 5 drink levels, 5 price levels). Participants completed 6 repetitions of each possible quantity/cost combination in a randomized order, yielding 150 total trials. Participants responded via key-press whether they would "drive" or "ride" or were indifferent to the choice on each trial before transitioning to the subsequent trial.

\section{Procedure}

This study used a single alcohol administration design, following alcohol administration procedures previously published by our laboratory (Amlung, McCarty, Morris, Tsai, \& McCarthy, 2015). Participants abstained from alcohol/drug use for 24 hours and refrained from eating for 1 hour prior to their lab sessions. Participants arrived at 10 a.m. and alcohol administration occurred at approximately noon. Sessions were conducted by two staff members. Staff members obtained informed consent and verified participant sobriety. Women were given a urine pregnancy test (no participants tested positive). To control for gastric content, participants were provided a small meal (15\% of recommended daily caloric intake based on body mass) 90 min prior to alcohol administration. Participants completed computerized questionnaires on 
demographics, alcohol and substance use history, and baseline AID measures prior to the alcohol administration.

Alcohol dose was calculated to produce a peak BrAC of $0.10 \mathrm{~g} \%$ at $60 \mathrm{~min}$ postconsumption based on biological sex, age, height, and weight (Curtin \& Fairchild, 2003). Participants were provided a 190-proof pure grain alcohol mixed drink with orange juice in a 1:3 ratio. Beverages were split into two glasses of equal volume. Participants consumed each beverage in 1 min, with a 5-min break in between.

Participants completed the previously described measures on their subjective intoxication, perceived dangerousness of driving, and willingness to drive at seven time points after alcohol consumption. Post-consumption measures were designed to capture specific, comparable BrACs on the ascending and descending limbs of the BrAC curve (BrACs targets: Time $2=0.03 \mathrm{~g} \%$, Time $3 / 8=0.06 \mathrm{~g} \%$, Time $4 / 7=0.08 \mathrm{~g} \%$, and Time $5 / 6=0.10 \mathrm{~g} \%$ ). Experimenters collected BrACs in 5-min intervals after alcohol consumption and administered questionnaires when BrAC targets were reached. Participants completed the intoxicated driving decision making task twice during the session at a $\mathrm{BrAC}$ target $=.07 / .07$, once on the ascending limb and once on the descending limb of the BrAC curve. The task lasted approximately $10 \mathrm{~min}$ at each administration.

Participants remained in the laboratory until their $\mathrm{BrAC}$ was below $0.04 \mathrm{~g} \%$. Transportation home was arranged and provided via a prepaid taxi or friend. Participants earned $\$ 15$ per hour for the alcohol administration session.

\section{Data Analytic Plan}

Participants were classified based on their decision making strategy, as described in the following section. The ecological validity of these classifications was tested using self-reported 
baseline measures, repeated measures under intoxication, and within-subjects analyses, as described below. All analyses were conducted in R (version 4.3) using lme4 (Bates, Maechker, Bolker, \& Walker, 2020) for the mixed effects models and JAGS (version 4.3) for the Bayesian cognitive modeling. Directional hypotheses, code, and data were preregistered and are available at: https://osf.io/zpd8j/.

\section{Bayesian Cognitive Modeling}

Our modeling approach implemented the methodology from McCarthy et al. (2021) which is an extension of the Bayesian change point method developed by Lee (2019). The core function of Lee's framework is to detect, under a set of sequential choices, when an individual's probability of selecting one alternative over another abruptly changes (i.e., change point). For example, an individual who employs a simple heuristic of always taking a ride service after drinking two or more alcoholic drinks would make very different AID choices at one beer than at 3 beers over the course of an evening. McCarthy et al. (2021) extended Lee (2019) by estimating change points for each individual across multiple choice conditions. McCarthy et al. defined different decision strategies by placing mathematical constraints on change points across conditions. This allows the classification of individuals based on whether their choices meaningfully change as a function of a specified decision factor, which is systematically varied across condition (e.g., multiple levels of ride service cost).

We used this modeling framework to estimate whether an individual's probability of choosing to take a ride service on our decision task was a function of either: (1) alcohol consumption level, or (2) alcohol consumption level and ride service cost. As an example of a single cost condition, let $y_{i, j}$ be the $i^{\text {th }}$ participant's response to a single ride service cost condition, under $j$-many drinks. Suppose a participant was repeatedly offered the choice to either drive at no 
cost or take a $\$ 20$ ride service, having consumed a specified number of drinks over a two-hour period. Suppose this participant consistently chooses to drive under this cost condition for all consumption levels of 3 or fewer drinks. Suppose also that this same individual, under the same $\$ 20$ cost condition, consistently chooses not to drive, for all consumption levels strictly greater than 3 drinks. Under this $\$ 20$ cost condition, this individual's change point would be 4 drinks. More formally, we model responses, $y_{i, j}$, as a multinomial random variable where the choice options are determined by their probabilities, $\theta_{\text {ride }}$ (choose ride service) and $\theta_{\text {drive }}$ (choose drive themselves) and $1-\theta_{\text {ride }}-\theta_{\text {drive }}$ (indifferent between riding and driving). The number of drinks at which an individual's ride and drive probabilities shift is denoted by $\tau$. For our example, the participant chooses to drive at a high probability for up to 3 drinks (e.g., $\theta_{\text {drive }}=.90 ; \theta_{\text {ride }}=.10$ ), with this probability dramatically changing at 4 drinks (e.g., $\left.\theta_{\text {drive }}=.10 ; \theta_{\text {ride }}=.90\right)$, eliciting a $\tau$ value of 4 drinks.

Following McCarthy et al. (2021) we define different decision strategies by modeling the relationships among change points for more than one cost condition. For a compensatory decision maker (i.e., cost sensitive), $\tau$ values are allowed to increase as ride service costs increase. In other words, the compensatory decision maker will require a higher consumption level as ride service costs increase before taking the safe ride service - see Figure 1. This decision strategy is operationalized within the model via the constraints: $\tau_{1} \leq \tau_{2} \leq \tau_{3} \leq \tau_{4} \leq \tau_{5}$, where $\tau_{\mathrm{k}}$ denotes the change point for the $k^{\text {th }}$ cost condition, ordered increasingly by ride service cost. For a non-compensatory decision maker (i.e., not cost sensitive), the $\tau$ value would be the same across all cost conditions and would have the following profile: $\tau_{1}=\tau_{2}=\tau_{3}=\tau_{4}=\tau_{5}$. This shows that the change point is consistent across all cost conditions. Such a decision maker is employing a simple rule that is not dependent on ride cost, e.g., "If I have more than 2 drinks, I 
always pay for the ride service." Finally, we considered an unconstrained model which places no constraints on the $\tau$ parameters. This model allows for any choice behavior and serves as a benchmark to compare the two substantive decision strategies. The posterior mean and mode were used to estimate the value of choice probabilities and change points. A spike and slab Bayesian prior (see e.g., Lee, 2019) was used over all possible $\tau$ values to determine whether a change point existed for each individual, and what value it should take for that ride service cost condition. Finally, Bayes factors were calculated using the product space method (Lodewyckx et al, 2011). We refer readers to McCarthy et al. (2021) and Lee (2019) for additional technical details.

Responses from each participant were classified according to the non-compensatory and compensatory models using Bayes factors separately for the ascending and descending limbs (Kass \& Raftery, 1995). We calculated two sets of Bayes factors for the decision making strategy used on each limb: (1) the non-compensatory model versus the unconstrained model, and (2) the compensatory model versus the unconstrained model. By the transitive property of Bayes factors, the Bayes factor of non-compensatory versus compensatory model is formed by taking the ratios of (1) and (2). Each participant's choice data was classified separately for each limb according to the model that attained the highest Bayes factor compared to the unconstrained model and had a Bayes factor of at least 1 against the next best performing model. Otherwise, that participant was labeled as 'unclassified' for that limb. Abbreviations are used in the text and figures to describe participants based on their decision strategies on the ascending and descending limbs in chronological order as: non-compensatory on the ascending limb - non-compensatory on the descending limb (denoted NN), non-compensatory - compensatory (NC), compensatory - 
compensatory $(\mathrm{CC})$, compensatory - non-compensatory $(\mathrm{CN})$. See Table 1 for a crosstabulation of decision making strategy use across both limbs.

\section{Analyses for Preregistered Directional Hypotheses}

Mixed effects models were conducted to examine whether decision making strategies on each limb were related to changes in subjective intoxication and perceived AID dangerousness across the BrAC curve. Fixed effects of ascending and descending limb classification, as well as their interaction, were assessed along with random slopes and intercepts for each participant over the time points. Time was assessed linearly and quadratically. Linear regressions were conducted to examine whether decision making strategies on each limb were related to differing levels of AID attitudes, intentions, and behavior. Main effects of ascending and descending limb classifications were assessed to determine whether compensatory and non-compensatory strategies were related to perceived danger of AID and the highest BrAC at which an individual was willing to drive. Interactions of ascending and descending limb classifications were also assessed to determine whether switching strategies from the ascending to descending limb were related to AID attitudes, intentions, and behavior.

\section{Results}

\section{Decision Making Classification under Intoxication}

We assessed whether individuals could be classified into two distinct decision making strategies and whether these classifications were stable or changed across limbs. These methods resulted in $73 \%$ of the sample being classified as either compensatory or non-compensatory on both limbs. Table 1 shows the number of individuals in each limb classification pattern along with the average change point for each classification pattern on the lowest (\$5) and highest (\$25) ride service cost condition. As shown in Table 1, on the limb when individuals used a non- 
compensatory strategy they, on average, had a consistent change point across ride service cost conditions on that same limb. When individuals used a compensatory strategy on a particular limb they had a change point that increased as the ride service cost increased at an average of $\$ 10$ for each additional drink on that same limb. There were no differences in observed BrAC based on decision strategy use (see Table 2); BrAC was equivalent for all strategy classifications when the decision task was administered across both the ascending (M[SD]: .071[.011]) and descending limb (M[SD]: .071[.010]).

\section{Intoxicated Perceptions based on Decision making Classification}

We assessed changes in subjective intoxication and perceived AID dangerousness at multiple timepoints across the ascending and descending limb across decision making classifications. We observed the same pattern across both subjective intoxication and perceived AID dangerousness across all decision making classifications. Both subjective intoxication and perceived AID dangerousness were highest on the ascending limb and began decreasing across the descending limb among all decision classification groups. This pattern is supported by the significant linear and quadratic effects of time for both subjective intoxication and perceived AID dangerousness (see Table 3). The limb decision classifications NN, CC, and CN reported similar levels of subjective intoxication and AID dangerousness across the ascending and descending limb. Significantly lower levels of subjective intoxication and AID dangerousness were reported across all timepoints for individuals who used a non-compensatory strategy on the ascending limb and switched to a compensatory strategy on the descending limb. This pattern is supported by a significant interaction between ascending classification and the quadratic effect of time for subjective intoxication (see Figure 2A) and AID dangerousness (see Figure 2B). This finding suggests that instability in decision strategy and switching to a cost sensitive 
(compensatory) strategy on the descending limb is associated with lower levels of subjective intoxication and perceptions of AID dangerousness, especially on the descending limb, which could lead to riskier decision making.

Similar to intoxicated perceptions of AID dangerousness, sober perceptions of AID dangerousness were also related to decision making strategies under intoxication. The lowest (i.e., the riskiest) sober perceptions of AID dangerousness were observed among those who switched from non-compensatory to compensatory strategy use in the decision task $(\mathrm{M}[\mathrm{SD}]=$ $1.89[.78])$ compared to those who were compensatory or non-compensatory on both limbs or switched from compensatory to non-compensatory strategy use (see Figure 3 and Table 4).

\section{AID Intentions and Behavior}

Compensatory strategy use on the descending limb was related to a greater willingness to drive at a higher $\mathrm{BrAC}(\mathrm{M}[\mathrm{SD}]=.09[.03])$ than those who were non-compensatory $(\mathrm{M}[\mathrm{SD}]=$ $.07[.04])$ on the descending limb (see Figure 4 and Table 4). Those using a compensatory strategy on the descending limb also reported greater intentions to drive while their observed BrAC was, on average, above the legal limit of .08. There were no significant main effects or interactions of decision making classification that predicted self-reported AID behavior in the past 3 months and 1 year $(p s>.05)$.

\section{Discussion}

Extending previous research on AID decision strategy use (McCarthy et al., 2021) and intoxicated decision making (Obst et al., 2018; George, Rogers, Duka, \& 2005; van Ravenzwaaij et al., 2012), we examined changes in decision making strategies under intoxication across the ascending and descending limbs of the BrAC curve. There was not a consistent effect of BrAC limb on decision strategies participants' used. While over a quarter of our sample changed 
strategy across limbs, participants were roughly equally likely to switch to a more risk averse strategy (i.e., non-compensatory) as to a riskier strategy (i.e., compensatory). However, results indicate that compensatory strategy use, especially on the descending limb, is associated with riskier AID attitudes and intentions. Switching to compensatory strategy use on the descending limb was related to lower subjective intoxication and lower perceived danger of AID. Those who used a compensatory strategy on the descending limb also reported being willing to drive at a BrAC that was, on average, above the legal limit.

Most individuals were consistent in their decision strategy, focusing solely on alcohol consumption (i.e., non-compensatory) or using trade-offs with alcohol consumption and ride service cost (i.e., compensatory). Those who maintained a consistent decision strategy also reported higher levels of subjective intoxication on both limbs. Consistent non-compensatory strategy users would drive after consuming, on average, 3 drinks in 2 hours, and would switch to using a ride service at higher drink numbers. Similarly, consistent compensatory strategy users chose to drive when drinking up to 3 drinks in 2 hours, but this was conditional on the cost of the ride service. On average, a $\$ 10$ increase in ride service was associated with a one drink increase in change point.

Understanding these decision strategies can inform interventions to encourage the use of AID alternatives when people are already intoxicated. Specifically, our results suggest tailoring AID intervention approaches to the decision strategies individuals use while intoxicated (DavisStober et al., 2019). Individuals who employ a compensatory strategy (i.e., ride service cost sensitive) may benefit more from interventions focused on reducing the cost of AID alternatives, such as free or discounted safe ride programs promoted at bars or on ride service apps (Fell et al., 2020). Although our results indicate that non-compensatory strategy users may, overall, be at 
reduced risk for AID, considerable variability was observed in the change point at which these decision makers switched to a ride service, with $50 \%$ and $60 \%$ of these participants choosing to drive after consuming 4 or more drinks in 2 hours on the ascending and descending limb, respectively. Based on our results, non-compensatory strategy users may not be as influenced by reductions in the cost of AID alternatives. Instead, these individuals may benefit from efforts aimed at reducing their drink limit, perhaps through just-in-time interventions that provide inthe-moment objective information about their BrAC level and AID risk at specific drink amounts.

\section{Limitations}

This research study has limitations in sample size, lack of a comparison or control group condition, and focuses solely on an early adulthood sample. Due to sample size limitations, some limb classification patterns had relatively fewer individuals than others. Lacking a control condition did not allow us to test whether intoxicated decision strategies align with sober decision strategies which could inform interventions before a drinking episode. Early adulthood has the highest rates of AID, but middle-to-older adults may have differing views on ride services and distinct patterns of acute alcohol tolerance which could better inform interventions among an older age group (Friedman et al., 2020; Conner et al., 2021). Although we were able to classify most individuals into either compensatory or non-compensatory strategy use, future research should determine whether there are other decision making strategies being used or the absence of identifiable strategies among unclassified individuals. Our assessment of decision making was limited to only AID and two common factors associated with AID decision making and not other contextual factors.

\section{Conclusions and Future Directions}


This study employed a laboratory alcohol administration protocol combined with Bayesian cognitive modeling to identify individuals' AID decision strategies under intoxication. Compensatory strategy use (i.e., sensitivity to the cost of a ride service), particularly on the descending limb, was associated with perceiving oneself to be less intoxicated, perceiving AID to be less dangerous, and being more willing to drive at BrACs above the legal limit. Future research is needed to understand what factors (e.g., subjective intoxication, AID attitudes, degree of acute tolerance) influence the use of the more risky compensatory strategy. In addition, future studies can explore the relative effectiveness of AID preventions strategies tailored to specific decision making strategies (e.g., reductions in the cost of AID alternatives for compensatory individuals). Finally, research is needed to assess these decision making strategies in more naturalistic drinking settings, as contextual factors (e.g., drinking location, social context) likely play a role in strategy use and the association between strategy use and real-world AID decisions. 


\section{References}

Amlung, M. T., Morris, D. H., \& McCarthy, D. M. (2014). Effects of acute alcohol tolerance on perceptions of danger and willingness to drive after drinking. Psychopharmacology, 231(22), 4271-4279. https://doi.org/10.1007/s00213$\underline{014-3579-1}$

Amlung, M., McCarty, K. N., Morris, D. H., Tsai, C. L., \& McCarthy, D. M. (2015). Increased behavioral economic demand and craving for alcohol following a laboratory alcohol challenge. Addiction, 110(9), 1421-1428. https://doi.org/10.1111/add.12897

Bates D., Mächler M., Bolker B., Walker S. (2015). Fitting linear mixed-effects models using lme4.” Journal of Statistical Software, 67(1), 1-48. 10.18637/jss.v067.i01.

Centers for Disease Control and Prevention (2015). Alcohol-impaired driving among U.S. adults. MorbMortWkly Rep Surveill Summ, 64: 814-7. Available at: https://www.cdc.gov/mmwr/preview/mmwrhtml/mm6430a2.htm

Conner, C. R., Ray, H. M., McCormack, R. M., Dickey, J. S., Parker, S. L., Zhang, X., Vera, R. M., Harvin, J. A., \& Kitagawa, R. S. (2021). Association of rideshare use with alcoholassociated motor vehicle crash trauma. JAMA Surgery, e212227. Advance online publication. https://doi.org/10.1001/jamasurg.2021.2227

Curtin, J. J., \& Fairchild, B. A. (2003). Alcohol and cognitive control: implications for regulation of behavior during response conflict. Journal of Abnormal Psychology, 112(3), 424-436. https://doi.org/10.1037/0021-843x.112.3.424

Davis-Stober, C. P., McCarty, K. N., \& McCarthy, D. M. (2019). Decision making and alcohol: health policy implications. Policy Insights from the Behavioral and Brain Sciences, 6(1), 64-71. 
Drew L., Royal D., Moulton B., Peterson A., Haddix D. (2008). National Survey of Drinking and Driving Attitudes and Behaviors. U.S. Department of Transportation, National Highway Traffic Safety Administration; Volume 1.

Fairlie, A. M., Quinlan, K. J., Dejong, W., Wood, M. D., Lawson, D., \& Witt, C. F. (2010). Sociodemographic, behavioral, and cognitive predictors of alcohol-impaired driving in a sample of U.S. college students. Journal of Health Communication, 15(2), 218-232. https://doi.org/10.1080/10810730903528074

Fell, J. C., Scolese, J., Achoki, T., Burks, C., Goldberg, A., \& DeJong, W. (2020). The effectiveness of alternative transportation programs in reducing impaired driving: A literature review and synthesis. Journal of Safety Research, 75, 128-139. https://doi.org/10.1016/j.jsr.2020.09.001

Friedman, J., Hendrix, V., Fustok, J., Reza, T., Madda, P., Smith, A., Mayer, S., Duchesne, J., Greiffenstein, P., \& Schroll, R. (2020). Correlation of ride sharing service availability and decreased alcohol-related motor vehicle collision incidence and fatality. The Journal of Trauma and Acute Care Surgery, 89(3), 441-447. https://doi.org/10.1097/TA.0000000000002802

George, S., Rogers, R. D., \& Duka, T. (2005). The acute effect of alcohol on decision making in social drinkers. Psychopharmacology, 182(1), 160-169. https://doi.org/10.1007/s00213$\underline{005-0057-9}$

Gigerenzer, G., \& Goldstein, D. G. (1996). Reasoning the fast and frugal way: models of bounded rationality. Psychological Review, 103(4), 650. 
Greene, K. M., Murphy, S. T., \& Rossheim, M. E. (2018). Context and culture: Reasons young adults drink and drive in rural America. Accident; Analysis and Prevention, 121, 194201. https://doi.org/10.1016/j.aap.2018.09.008

Greenfield, T. K., \& Rogers, J. D. (1999). Alcoholic beverage choice, risk perception and selfreported drunk driving: effects of measurement on risk analysis. Addiction, 94(11), 17351743. https://doi.org/10.1046/j.1360-0443.1999.9411173510.x

Grube, J. W., \& Voas, R. B. (1996). Predicting underage drinking and driving behaviors. Addiction, 91(12), 1843-1857. https://doi.org/10.1046/j.1360-0443.1996.911218438.x

Gustin, J. L., \& Simons, J. S. (2008). Perceptions of level of intoxication and risk related to drinking and driving. Addictive Behaviors, 33(4), 605-615. https://doi.org/10.1016/j.addbeh.2007.11.010

Jonah B. A. (1997). Sensation seeking and risky driving: a review and synthesis of the literature. Accident; Analysis and Prevention, 29(5), 651-665. https://doi.org/10.1016/s0001$\underline{4575(97) 00017-1}$

Kass, R. E., \& Raftery, A. E. (1995). Bayes factors. Journal of the American Statistical Association, 90(430), 773-795.

Lee, M. D. (2019). A simple and flexible Bayesian method for inferring step changes in cognition. Behavior Research Methods, 51(2), 948-960.

Lee, M. D., Gluck, K. A., \& Walsh, M. M. (2019). Understanding the complexity of simple decisions: Modeling multiple behaviors and switching strategies. Decision, 6(4), 335368. https://doi.org/10.1037/dec0000105 
Lodewyckx, T., Kim, W., Lee, M. D., Tuerlinckx, F., Kuppens, P., \& Wagenmakers, E. J. (2011). A tutorial on Bayes factor estimation with the product space method. Journal of Mathematical Psychology, 55(5), 331-347.

Uber \& Mothers Against Drunk Driving. (2015). More options. Shifting mindsets. Driving better choices [internet]. Available at: http://blog.uber.com/uber-madd.

McCarthy, D. M., Pedersen, S. L., Thompsen, D. M., \& Leuty, M. E. (2006). Development of a measure of drinking and driving expectancies for youth. Psychological Assessment, 18(2), 155-164. https://doi.org/10.1037/1040-3590.18.2.155

McCarthy, D. M., McCarty, K. N., Hatz, L. E., Prestigiacomo, C. J., Park, S., \& Davis-Stober, C. P. (2021). Applying Bayesian cognitive models to decisions to drive after drinking. Addiction, 116(6), 1424-1430. https://doi.org/10.1111/add.15302

Mecca, J. T., Medeiros, K. E., Giorgini, V., Gibson, C., Mumford, M. D., Connelly, S., \& Devenport, L. D. (2014). The influence of compensatory strategies on ethical decision making. Ethics \& Behavior, 24(1), 73-89.

Motschman, C. A., Warner, O. M., Wycoff, A. M., Davis-Stober, C. P., \& McCarthy, D. M. (2020a). Context, acute tolerance, and subjective response affect alcohol-impaired driving decisions. Psychopharmacology, 237(12), 3603-3614. https://doi.org/10.1007/s00213-020-05639-0

Motschman, C. A., Hatz, L. E., McCarty, K. N., Merkle, E. C., Trull, T. J., \& McCarthy, D. M. (2020b). Event-Level Predictors of Alcohol-Impaired Driving Intentions. Journal of Studies on Alcohol and Drugs, 81(5), 647-654. https://doi.org/10.15288/jsad.2020.81.647 
National Highway Traffic Safety Administration (NHTSA). (2020). State alcohol impaireddriving estimates: 2018 data (Traffic Safety Facts. Report No. DOT HS 812 917). National Center for Statistics and Analysis.

Newell, B. R., Weston, N. J., \& Shanks, D. R. (2003). Empirical tests of a fast-and-frugal heuristic: Not everyone "takes-the-best". Organizational Behavior and Human Decision Processes, 91(1), 82-96.

Obst, E., Schad, D. J., Huys, Q. J., Sebold, M., Nebe, S., Sommer, C., Smolka, M. N., \& Zimmermann, U. S. (2018). Drunk decisions: Alcohol shifts choice from habitual towards goal-directed control in adolescent intermediate-risk drinkers. Journal of Psychopharmacology, 32(8), 855-866. https://doi.org/10.1177/0269881118772454

Ouimet, M. C., Brown, T. G., Corado, L., Paquette, M., \& Robertson, R. D. (2020). The effects of alcohol dose, exposure to an in-vehicle alcohol feedback device, and subjective responses to alcohol on the decision to drink-drive in young drivers. Accident; Analysis and Prevention, 139, 105495. https://doi.org/10.1016/j.aap.2020.105495

Lipari, R.N., Hughes, A. and Bose, J. (2016). Driving under the influence of alcohol and illicit drugs. The CBHSQ Report. Center for Behavioral Health Statistics and Quality, Substance Abuse and Mental Health Services Administration, Rockville, MD.

Schweizer, T. A., Jolicoeur, P., Vogel-Sprott, M., \& Dixon, M. J. (2004). Fast, but error-prone, responses during acute alcohol intoxication: effects of stimulus-response mapping complexity. Alcoholism, Clinical and Experimental Research, 28(4), 643-649. https://doi.org/10.1097/01.alc.0000121652.84754.30

Schweizer, T. A., \& Vogel-Sprott, M. (2008). Alcohol-impaired speed and accuracy of cognitive functions: a review of acute tolerance and recovery of cognitive performance. 
Experimental and Clinical Psychopharmacology, 16(3), 240-250.

https://doi.org/10.1037/1064-1297.16.3.240

Sloan, F. A., McCutchan, S. A., \& Eldred, L. M. (2017). Alcohol-Impaired Driving and Perceived Risks of Legal Consequences. Alcoholism, Clinical and Experimental Research, 41(2), 432-442. https://doi.org/10.1111/acer.13298

Treloar, H. R., \& McCarthy, D. M. (2012). Effects of mood and urgency on activation of general and specific alcohol expectancies. Addictive Behaviors, 37(1), 115-118. https://doi.org/10.1016/j.addbeh.2011.07.006

Turrisi, R., \& Jaccard, J. (1992). Cognitive and attitudinal factors in the analysis of alternatives to drunk driving. Journal of Studies on Alcohol, 53(5), 405-414. https://doi.org/10.15288/jsa.1992.53.405

van Ravenzwaaij, D., Dutilh, G., \& Wagenmakers, E. J. (2012). A diffusion model decomposition of the effects of alcohol on perceptual decision making. Psychopharmacology, 219(4), 1017-1025. https://doi.org/10.1007/s00213-011-2435-9

Von Gunten, C. D., \& Scherer, L. D. (2019). Self-other differences in multiattribute decision making: Compensatory versus noncompensatory decision strategies. Journal of Behavioral Decision Making, 32(2), 109-123.

Weafer, J., \& Fillmore, M. T. (2012). Acute tolerance to alcohol impairment of behavioral and cognitive mechanisms related to driving: drinking and driving on the descending limb. Psychopharmacology, 220(4), 697-706. https://doi.org/10.1007/s00213-011-2519-6 


\section{TABLES}

Table 1. Average number of drinks for each cost level by classification group across limbs

\begin{tabular}{cc|c|cc|cc}
\hline & & \multicolumn{2}{c}{ Ascending Limb } & \multicolumn{2}{c}{ Descending Limb } \\
\cline { 5 - 7 } $\begin{array}{c}\text { Ascending } \\
\text { Classification }\end{array}$ & $\begin{array}{c}\text { Descending } \\
\text { Classification }\end{array}$ & $\mathbf{N}$ & $\mathbf{\$ 5}$ & $\mathbf{\$ 2 5}$ & $\mathbf{\$ 5}$ & $\mathbf{\$ 2 5}$ \\
\hline Non-Compensatory & Non-Compensatory & 21 & $2.66[1.08]$ & $2.71[1.03]$ & $2.81[1.10]$ & $2.81[.96]$ \\
Non-Compensatory & Compensatory & 9 & $3.00[1.00]$ & $3.44[1.11]$ & $3.11[.89]$ & $4.78[1.12]$ \\
Compensatory & Non-Compensatory & 7 & $2.57[.49]$ & $4.86[1.12]$ & $3.14[.99]$ & $3.86[.83]$ \\
Compensatory & Compensatory & 21 & $2.66[.71]$ & $4.81[1.01]$ & $2.67[.71]$ & $4.86[1.08]$ \\
\hline \hline
\end{tabular}

Means with standard deviation in brackets are listed above for the average change point in each decision strategy group.

Table 2. Differences in BrAC across study timepoints and by decision making classification

\begin{tabular}{|c|c|c|c|}
\hline \multirow[b]{2}{*}{ Predictors } & \multicolumn{3}{|c|}{ BrAC } \\
\hline & Estimates & $C I$ & $p$ \\
\hline Intercept & 3.52 & $2.76-4.29$ & $<0.001$ \\
\hline Timepoint & 2.68 & $2.35-3.01$ & $<0.001$ \\
\hline Ascending & 0.15 & $-1.24-1.55$ & 0.828 \\
\hline Descending & -0.88 & $-2.41-0.65$ & 0.260 \\
\hline Timepoint $^{2}$ & -0.32 & $-0.36--0.28$ & $<0.001$ \\
\hline Timepoint $*$ Ascending & -0.17 & $-0.77-0.44$ & 0.588 \\
\hline
\end{tabular}




$\begin{array}{lccc}\text { Timepoint * Descending } & 0.19 & -0.48-0.85 & 0.584 \\ \text { Ascending * Descending } & 1.54 & -0.53-3.61 & 0.146 \\ \text { Descending * Timepoint }^{2} & -0.00 & -0.09-0.07 & 0.743 \\ \text { Ascending * Timepoint }^{2} & 0.02 & -0.05-0.10 & 0.544 \\ \text { Timepoint * Ascending * Descending }^{*} & -0.47 & -1.37-0.42 & 0.302 \\ \text { Timepoint }^{2} \text { * Ascending * Descending } & 0.05 & -0.06-0.16 & 0.378\end{array}$

\section{Random Effects}

\begin{tabular}{ll}
$\sigma^{2}$ & 0.75 \\
$\tau_{00 \text { subject }}$ & 1.38 \\
$\mathrm{~N}_{\text {subject }}$ & 58 \\
\hline Observations & 406 \\
Marginal $\mathrm{R}^{2}$ & 0.613
\end{tabular}

Table 3. Decision making classification differences in subjective intoxication and perceived AID dangerousness across study timepoints

\begin{tabular}{lcccccc} 
& \multicolumn{3}{c}{ Subjective Intoxication } & & \multicolumn{3}{c}{ Perceived AID Dangerousness } \\
Predictors & Estimates & $C I$ & $p$ & Estimates & CI & p \\
\hline Intercept & 27.39 & $13.12-41.66$ & $<\mathbf{0 . 0 0 1}$ & 28.45 & $11.46-45.44$ & $\mathbf{0 . 0 0 1}$ \\
Timepoint & 11.80 & $7.40-16.20$ & $<\mathbf{0 . 0 0 1}$ & 12.18 & $7.47-16.89$ & $<\mathbf{0 . 0 0 1}$ \\
Ascending & -7.87 & $-33.04-17.30$ & 0.540 & 1.76 & $-28.21-31.72$ & 0.909 \\
Descending & -5.82 & $-33.32-21.68$ & 0.678 & 1.75 & $-31.98-34.49$ & 0.916
\end{tabular}




\begin{tabular}{|c|c|c|c|c|c|c|}
\hline Timepoint $^{2}$ & -1.92 & $-2.42--1.42$ & $<0.001$ & -1.92 & $-2.46--1.39$ & $<0.001$ \\
\hline Timepoint $*$ Ascending & -6.63 & $-14.40-1.14$ & 0.094 & -10.88 & $-19.19--2.57$ & 0.010 \\
\hline Timepoint $*$ Descending & 5.25 & $-3.24-13.74$ & 0.225 & 2.27 & $-6.81-11.35$ & 0.624 \\
\hline Ascending $*$ Descending & 19.14 & $-17.88-56.16$ & 0.311 & 6.27 & $-37.80-50.34$ & 0.780 \\
\hline Descending $*$ Timepoint $^{2}$ & -0.59 & $-1.56-0.38$ & 0.233 & -0.35 & $-1.38-0.68$ & 0.508 \\
\hline Ascending $*$ Timepoint $^{2}$ & 0.89 & $0.00-1.77$ & 0.050 & 1.23 & $0.28-2.17$ & 0.011 \\
\hline Timepoint $*$ Ascending $*$ Descending & -2.35 & $-13.77-9.07$ & 0.687 & 3.11 & $-9.11-15.34$ & 0.618 \\
\hline Timepoint $^{2} *$ Ascending $*$ Descending & 0.12 & $-1.18-1.42$ & 0.858 & -0.28 & $-1.67-1.12$ & 0.697 \\
\hline \multicolumn{7}{|l|}{ Random Effects } \\
\hline$\sigma^{2}$ & \multicolumn{4}{|c|}{104.57} & & 119.82 \\
\hline$\tau_{00 \text { subject }}$ & \multicolumn{4}{|c|}{752.34} & & 1141.10 \\
\hline$\tau_{11 \text { subject.timepoint }}$ & \multicolumn{4}{|c|}{12.48} & & 14.55 \\
\hline$\rho_{01 \text { subject }}$ & \multicolumn{4}{|c|}{-0.67} & & -0.70 \\
\hline ICC & \multicolumn{4}{|c|}{0.82} & & 0.86 \\
\hline $\mathrm{N}_{\text {subject }}$ & \multicolumn{4}{|c|}{56} & & 56 \\
\hline Observations & \multicolumn{4}{|c|}{392} & \multicolumn{2}{|r|}{390} \\
\hline Marginal $\mathrm{R}^{2}$ / Conditional $\mathrm{R}^{2}$ & \multicolumn{4}{|c|}{$0.180 / 0.854$} & \multicolumn{2}{|c|}{$0.136 / 0.876$} \\
\hline
\end{tabular}

$\underline{\text { Table 4. Differences in AID Danger and Willingness to Drive by Ascending and Descending Limb Classification }}$

Baseline Danger

Highest Driving BrAC

\begin{tabular}{lllllll} 
Predictors & Estimates & $C I$ & $p$ & Estimates & $C I$ & $p$ \\
\hline
\end{tabular}




\begin{tabular}{lrrr|rrrr} 
(Intercept) & 2.86 & $2.48-3.24$ & $\mathbf{< 0 . 0 0 1}$ & .082 & $0.07-0.10$ & $<\mathbf{0 . 0 0 1}$ \\
Ascending & -0.97 & $-1.66--0.28$ & $\mathbf{0 . 0 0 7}$ & .009 & $-0.02-0.04$ & .547 \\
Descending & -0.43 & $-1.19-0.33$ & 0.261 & -.034 & $-0.06--0.003$ & $\mathbf{. 0 3 4}$ \\
Ascending * Descending & 1.06 & $0.04-2.09$ & $\mathbf{0 . 0 4 2}$ & .016 & $-0.03-0.06$ & .456 \\
\hline Observations & & 58 & & & \\
$\mathrm{R}^{2} / \mathrm{R}^{2}$ adjusted & & $0.130 / 0.081$ & & & & \\
\end{tabular}


FIGURES

Figure 1. Change points for two distinct decision strategies

Change Points for Two Hypothetical Individuals

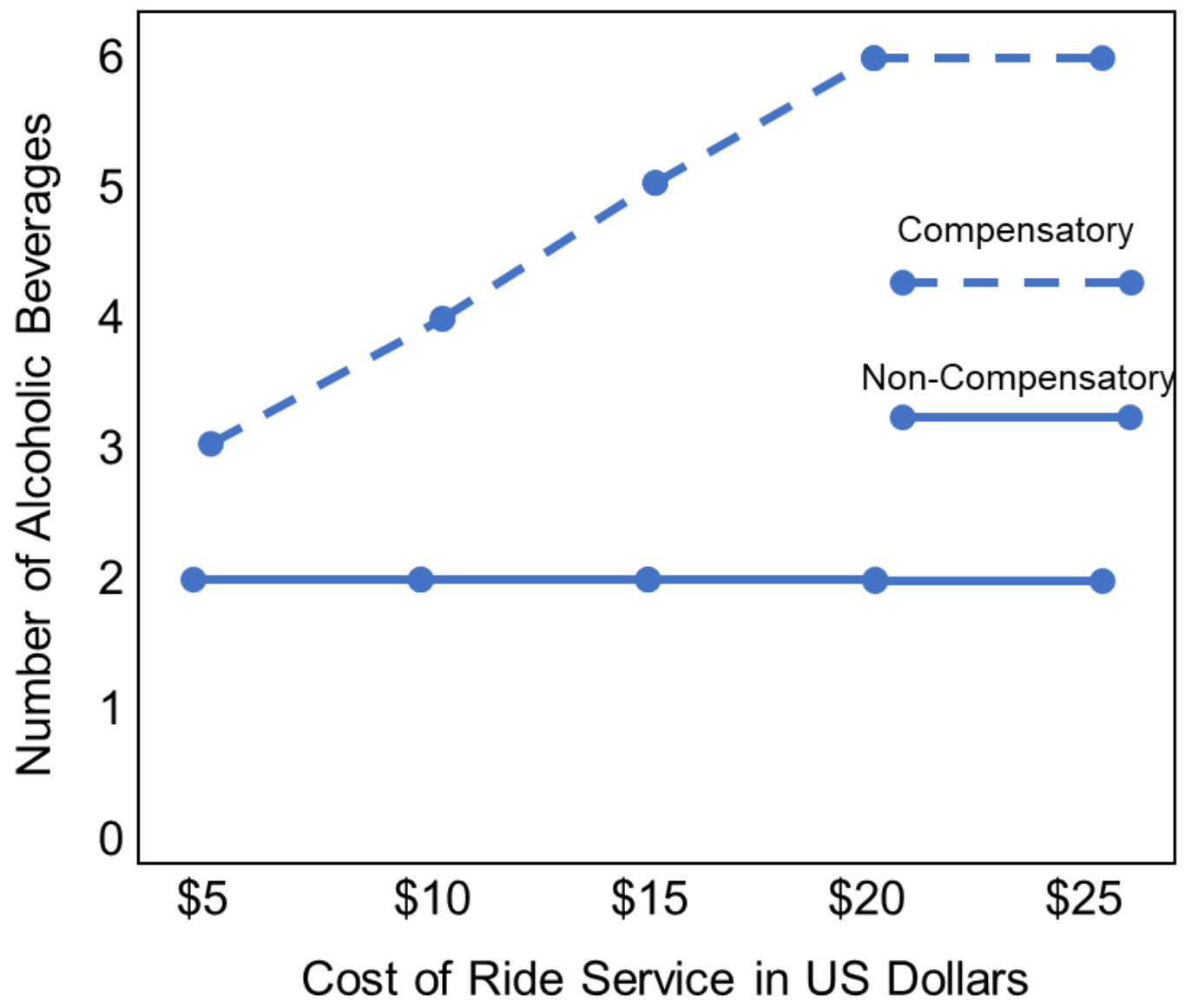


Figure 2. Subjective intoxication (A) AID dangerousness (B) across all study timepoints by classification group
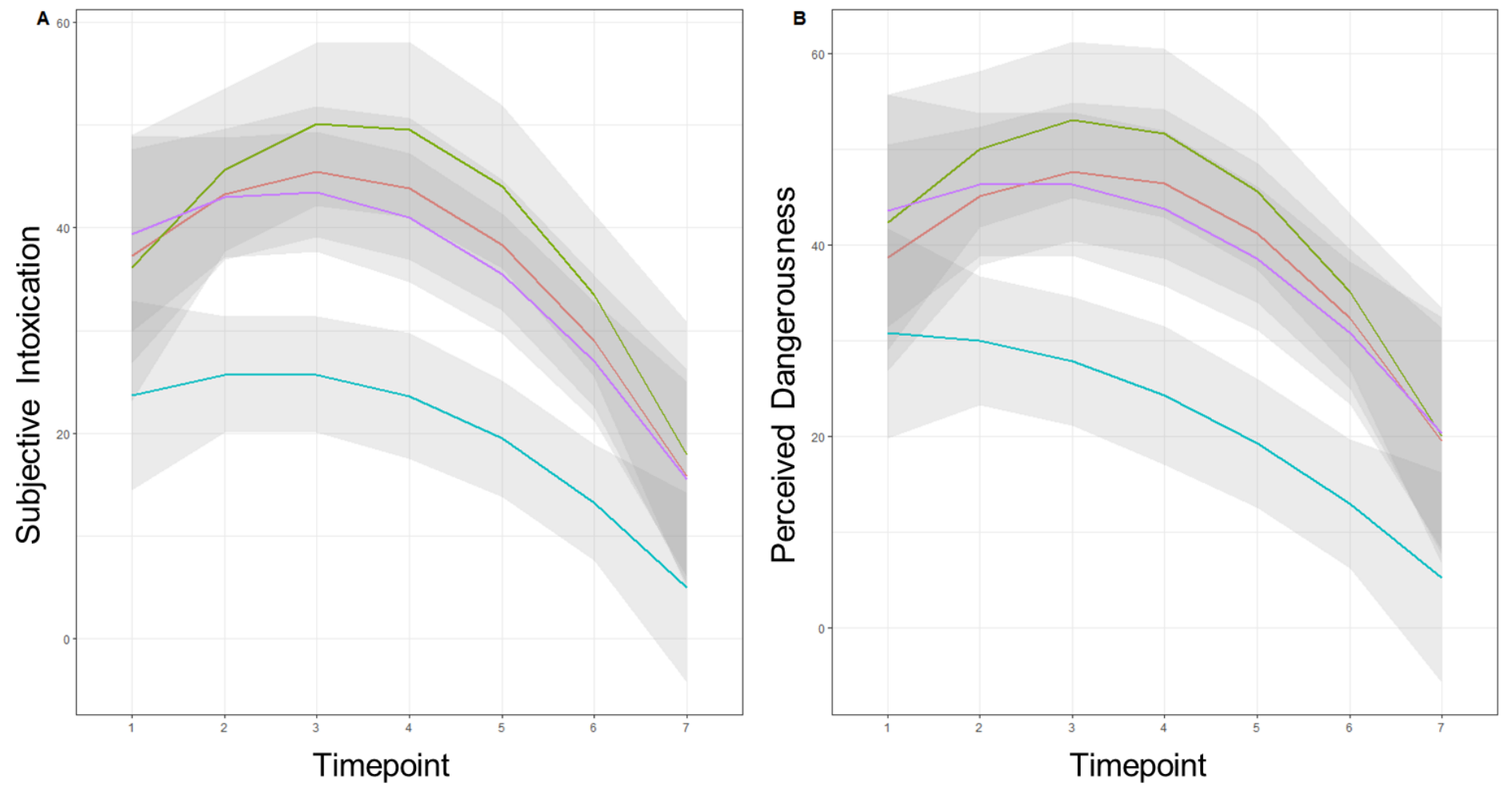

\section{Classification}

- Compensatory - Compensatory

- Compensatory - Non-Compensatory

- Non-Compensatory - Compensatory

- Non-Compensatory - Non-Compensatory 
Figure 3. Perceptions of alcohol-impaired driving dangerousness by classification group

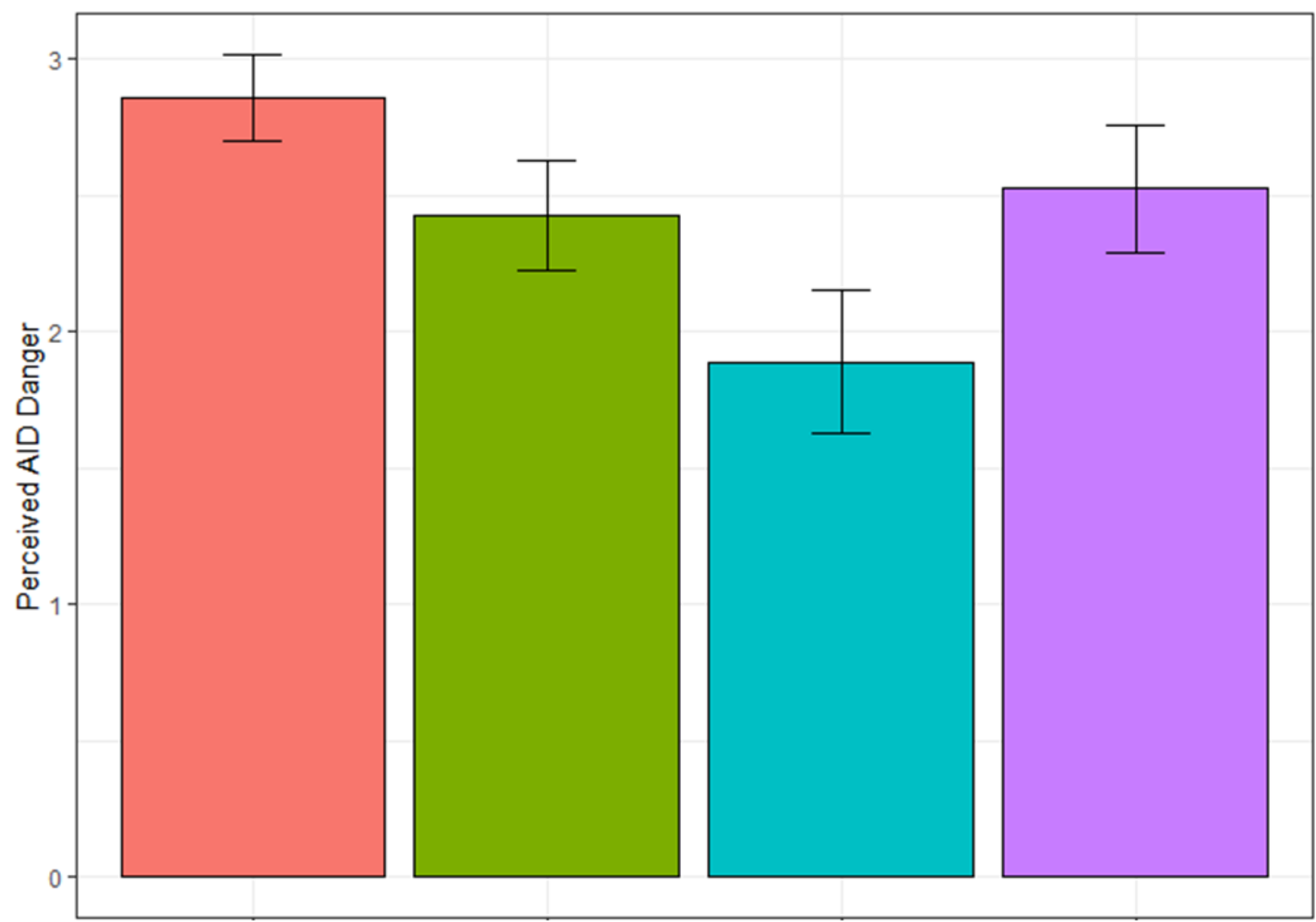

Classification

Compensatory - Compensatory Compensatory - Non-Compensatory Non-Compensatory - Compensatory Non-Compensatory - Non-Compensatory 
Figure 4. Willingness to drive under intoxication by classification group

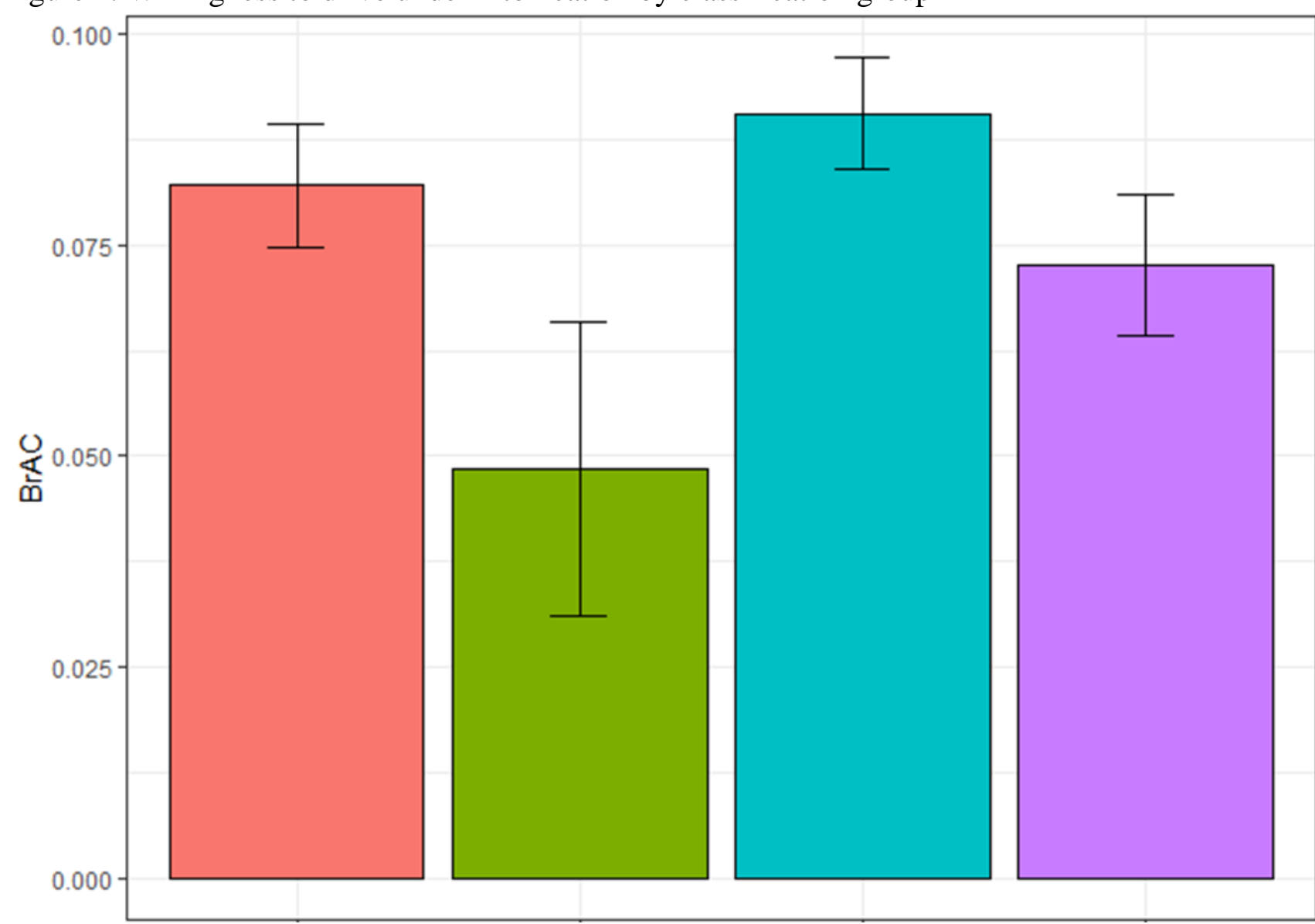

Classification

$\square$ Compensatory - Compensatory Compensatory - Non-Compensatory Non-Compensatory - Compensatory Non-Compensatory - Non-Compensatory 